Journal of Medical Genetics, 1983, 20, 236-239

La Pratique de l'Analyse Chromosomique

By B Dutrillaux and J Couturier. (Pp 87; figures.) Masson: Paris. 1981.

This small booklet, wrtten in French, is the twelfth publication in the series 'Techniques de Laboratoire'. It consists of detailed descriptions of techniques and procedures routinely applied in cytogenetics laboratories. It is a good bench manual, especially useful to beginners in the field of human chromosome studies.

M SeABright

\section{The Genetics of Neurological Disorders}

Oxford Monographs on Medical Genetics No 9. By Michael Baraitser. (Pp xii +516 ; figures + tables. £35.00.) Oxford: Oxford University Press. 1982.

This book is a member of the excellent series of Oxford monographs on medical genetics and succeeds one of the same title by Dr R T C Pratt, who, in 1967, was one of the first to put some order into neurological genetics and whose book was both comprehensive and interesting. Since the publication of that book there have been great advances in the delineation of neurological genetic entities. At the same time, neurology has changed from a largely clinical discipline to one where scientific techniques predominate. For example, the use of histochemical stains and electron microscopy has revolutionised the classification of some muscular dystrophies, even causing some temporary confusion, since disorders described at the beginning of the century are reclassified if there are living descendants available for muscle biopsy! Also, the use of CT scanning has enlarged our knowledge of, and ability to recognise, disorders associated with cerebral malformations. Dr Baraitser is a good choice to undertake the onerous burden of updating The Genetics of Neurological Disorders. He runs a genetic counselling service at the National Hospital for Nervous Diseases and is therefore familiar with the questions patients and their families ask, and he is Consultant Clinical Geneticist at The Hospital for Sick Children, and is therefore experienced in the recognition of syndromes.

Dr Baraitser has kept largely to the order and content of Pratt's earlier book but there are additional chapers and some have been expanded. The subjects covered include abnormalities and syndromes of the cranial nerves; malformations of $\frac{\bar{\sigma}}{\bar{D}}$ the skull, spine, and nervous system; dementia; $\underset{ه}{\overparen{ }}$ epilepsy; tumours; phakomatoses; and static and $\stackrel{\circ}{2}$ degenerative disorders of the basal ganglia, cere- $\infty$ bellum, cerebellar pathways, spinal cord, peripheral $\overrightarrow{0}$ nerves, and muscle. A paediatric neurologist will also find useful the sections on dyslexia, cerebral $\vec{\omega}$ palsy, and degenerative disorders of childhood. The genetics of mental subnormality and psychiatric disorders have been dealt with in an earlier volume in the series.

Dr Baraitser states in the preface that "The main purpose of this book is to help with the identification $\mathscr{\omega}^{\circ}$ of these (neurological) syndromes and to assist the $\frac{\text { ? }}{5}$ clinician in the derivation of risks of recurrence". $\mathrm{He}-$ succeeds well in these aims, particularly regarding the $\overleftarrow{c}$ identification of syndromes. He has reviewed more neurological disorders than I care to think of: about $\vec{\bullet}$ the sum of reported experience of neurologists for a century or so! Many of these disorders have occurred in only a single family; the listing of them provides a comprehensive source of reference for clinicians struggling to classify an unusual constellation of signs. Empirical risks for genetic counselling are usually given, but there remains some uncertainty on how to counsel an isolated case. For example, how often is scapuloperoneal muscular dystrophy genetic and how often non-genetic? What is the chance that a male infant, severely ill with centronuclear myopathy, has an X linked variety? Although not stated in the preface, I consider this book to be directed largely to neurologists, for the clinical criteria by which a particular diagnosis is reached are not always given and many of the conditions are so esoteric that they are unlikely to come first to a clinical geneticist.

I was disappointed not to find Dr Baraitser's opinions on some controversial issues in this book. For example, I should like to have known his opinions on the following. What is the relative frequency of mutation in Duchenne muscular dystrophy in the two sexes? What proportion of patients with Huntington's chorea are new mutants? What is N the explanation of the frequent paternal descent in juvenile Huntington's chorea, and the maternal descent in neonatal myotonic dystrophy? Is the predisposition to Parkinson's disease the result of $\stackrel{9}{?}$ monogenic, polygenic, or oligogenic inheritance? 0 These topics are either not discussed or else the various opinions from different workers are listed. I would have welcomed Dr Baraitser's own interpretation of some of these issues. 